\title{
Convergence of linking Baskakov-type operators
}

\author{
Ulrich Abel $^{1}$ (D) Margareta Heilmann ${ }^{2}$ (D) Vitaliy Kushnirevych ${ }^{1}$
}

Published online: 26 March 2020

(c) The Author(s) 2020

\begin{abstract}
In this paper we consider a link $B_{n, \rho}$ between Baskakov type operators $B_{n, \infty}$ and genuine Baskakov-Durrmeyer type operators $B_{n, 1}$ depending on a positive real parameter $\rho$. The topic of the present paper is the pointwise limit relation $\left(B_{n, \rho} f\right)(x) \rightarrow\left(B_{n, \infty} f\right)(x)$ as $\rho \rightarrow \infty$ for $x \geq 0$. As a main result we derive uniform convergence on each compact subinterval of the positive real axis for all continuous functions $f$ of polynomial growth.
\end{abstract}

Keywords Approximation by positive operators $\cdot$ Rate of convergence $\cdot$ Degree of approximation

Mathematics Subject Classification 41A36 - 41A25

\section{Introduction}

The so-called Baskakov type operators depending on a parameter $c \in \mathbb{R}$ were defined by Baskakov [1]. They are suitable for the approximation of functions being continuous on the underlying interval. It is well known that for the special choice of $c=-1, c=0$ and $c=1$, respectively, one derives the classical Bernstein, Szász-Mirakjan and Baskakov operators, respectively.

In the context of approximation of integrable functions the Baskakov-Durrmeyer type operators play an important role. The construction is based on the corresponding definition of Bernstein-Durrmeyer operators (see $[3,6,8,9,14]$ for further definitions). Although they have outstanding properties, i.e., they commute and are self-adjoint, they don't interpolate at finite endpoints of the interval and they only preserve constants. If the functions have

$\bowtie$ Ulrich Abel

Ulrich.Abel@mnd.thm.de

Margareta Heilmann

heilmann@math.uni-wuppertal.de

Vitaliy Kushnirevych

Vitaliy.Kushnirevych@mnd.thm.de

1 Department MND, Technische Hochschule Mittelhessen, Wilhelm-Leuschner-Straße 13, 61169 Friedberg, Germany

2 School of Mathematics and Natural Sciences, University of Wuppertal, Gaußstraße 20, 42119 Wuppertal, Germany 
supplemental finite limits at finite endpoints of the interval a modification of the BaskakovDurrmeyer type operators leads to the genuine variants which, like the classical operators, preserve all linear functions and interpolate at finite endpoints of the interval.

During the last years the question arose how the classical operators and their genuine counterparts are connected. This investigation was initiated by Păltănea $[10,11]$ by introducing a non-trivial link between Bernstein and Szász-Mirakjan operators, respectively, and their genuine Durrmeyer modifications (see also [4,5,12]).

The topic of the present paper is the investigation of convergence properties of linking Baskakov-Durrmeyer type operators for $c>0$ when the linking parameter $\rho$ tends to infinity.

Let $c, n, v, \rho \in \mathbb{R}$, such that $n>c \geq 0, \rho>0, v \geq 0$, and $x \in[0, \infty)$. Then the basis functions are given by

$$
p_{n, v}^{[c]}(x)= \begin{cases}\frac{(n x)^{v}}{v !} e^{-n x}, & \text { if } c=0, \\ \frac{\Gamma\left(\frac{n}{c}+v\right)}{\Gamma\left(\frac{n}{c}\right) \Gamma(v+1)}(c x)^{v}(1+c x)^{-n / c-v}, & \text { if } c>0 .\end{cases}
$$

It can be shown that $\lim _{c \rightarrow 0^{+}} p_{n, v}^{[c]}(x)=p_{n, v}^{[0]}(x)$ for each $x \geq 0$. Using the Euler beta function $B(x, y)=\Gamma(x) \Gamma(y) / \Gamma(x+y)$ for $x, y>0$, we obtain in case $c>0$ the representation

$$
(n-c) \cdot p_{n, v}^{[c]}(x)=\frac{c}{B(n / c-1, v+1)}(c x)^{v}(1+c x)^{-n / c-v} .
$$

For the sake of brevity and in order to be consistent with notations in several references, we put throughout the paper

$$
\mu_{n, v, \rho}^{[c]}(x)=(n \rho+c) p_{n \rho+2 c, v \rho-1}^{[c]}(x)
$$

for $v \geq 1 / \rho$. For $c>0$, we have the explicit representation

$$
\mu_{n, v, \rho}^{[c]}(x)=\frac{c}{B\left(\frac{n \rho}{c}+1, v \rho\right)}(c x)^{v \rho-1}(1+c x)^{-\frac{n \rho}{c}-v \rho-1} .
$$

It is well known that $(n-c) \int_{0}^{\infty} p_{n, v}^{[c]}(t) d t=1$ for all $v \geq 0$, so, obviously

$$
\int_{0}^{\infty} \mu_{n, v, \rho}^{[c]}(t) d t=1 \quad(v \geq 1 / \rho) .
$$

In the following definition we assume that $f:[0, \infty) \rightarrow \mathbb{R}$ is given in such a way that the corresponding integrals and series are convergent.

Definition 1 Let $c \geq 0$. The operators of Baskakov-type are defined by

$$
\left(B_{n, \infty}^{[c]} f\right)(x)=\sum_{\nu=0}^{\infty} p_{n, \nu}^{[c]}(x) f\left(\frac{\nu}{n}\right),
$$

and the genuine Baskakov-Durrmeyer type operators are denoted by

$$
\left(B_{n, 1}^{[c]} f\right)(x)=p_{n, 0}^{[c]}(x) f(0)+\sum_{\nu=1}^{\infty} p_{n, v}^{[c]}(x) \int_{0}^{\infty} \mu_{n, v, 1}^{[c]}(t) f(t) d t .
$$


Depending on a parameter $\rho>0$ the linking operators are given by

$$
\left(B_{n, \rho}^{[c]} f\right)(x)=p_{n, 0}^{[c]}(x) f(0)+\sum_{\nu=1}^{\infty} p_{n, \nu}^{[c]}(x) \int_{0}^{\infty} \mu_{n, \nu, \rho}^{[c]}(t) f(t) d t .
$$

Note that the genuine Baskakov-Durrmeyer type operators (1.2) are usually defined in the more explicit form

$$
\left(B_{n, 1}^{[c]} f\right)(x)=p_{n, 0}^{[c]}(x) f(0)+(n+c) \sum_{\nu=1}^{\infty} p_{n, \nu}^{[c]}(x) \int_{0}^{\infty} p_{n+2 c, \nu-1}^{[c]}(t) f(t) d t .
$$

Setting $c=0$ in (1.2) leads to the Phillips operators [13], the case $c>0$ was investigated in [15]. To the best of our knowledge, the case $c=0$ in (1.3) was first considered in [11] and the general case in [7].

The purpose of this paper is to prove the $\operatorname{limit}_{\rho \rightarrow \infty}\left(B_{n, \rho}^{[c]} f\right)(x)=\left(B_{n, \infty}^{[c]} f\right)(x)$ for all continuous functions $f$ on $[0, \infty)$ of polynomial growth. In the following theorem we state our main result.

Theorem 1.1 Let c, $\gamma>0$. Assume that $f \in C[0, \infty) \rightarrow \mathbb{R}$ satisfies the growth condition $f(t)=O\left(t^{\gamma}\right)$ as $t \rightarrow \infty$. Then, for any $b>0$, there is a constant $\rho_{0}>0$ such that $B_{n, \rho} f$ exists for all $\rho \geq \rho_{0}$ and

$$
\lim _{\rho \rightarrow \infty}\left(B_{n, \rho}^{[c]} f\right)(x)=\left(B_{n, \infty}^{[c]} f\right)(x)
$$

uniformly for $x \in[0, b]$.

The case $c=0$ was solved by Păltănea [12, Theorem 4] for functions $f \in C[0, \infty)$ satisfying $f(t)=O\left(e^{\gamma t}\right)$ as $t \rightarrow \infty$, where $\gamma$ is an arbitrary positive constant.

Further results concerning the limit of the operators $B_{n, \rho}$ as $\rho \rightarrow \infty$ for $c \geq 0$ were proved in [2,7]. From the explicit representations of the images of polynomials for all operators $B_{n, \rho}$ it was possible to derive immediately that for $c \geq 0$

$$
\lim _{\rho \rightarrow \infty}\left(B_{n, \rho}^{[c]} p\right)(x)=\left(B_{n, \infty}^{[c]} p\right)(x)
$$

uniformly on every compact subinterval of $[0, \infty)$ for each polynomial $p$ (see $[7$, Theorem 1, Theorem 2, Corollary 1]).

A different function space was considered in [2] for the case $c \geq 0$. For $f \in C^{2}[0, \infty)$ with $\left\|f^{\prime \prime}\right\|_{\infty}<\infty$

$$
\lim _{\rho \rightarrow \infty}\left(B_{n, \rho}^{[c]} f\right)(x)=\left(B_{n, \infty}^{[c]} f\right)(x)
$$

uniformly on every compact subinterval of $[0, \infty)$ (see [2, Lemma 5 , Corollary 3$]$ ).

\section{Auxiliary results}

In this section we present several lemmata which are needed for the proof of our main result in Sect. 3.

Let $W[0, \infty)$ be the class of all locally integrable functions on $[0, \infty)$ of polynomial growth, which are bounded on each compact subinterval of $[0, \infty)$. Obviously, for every $c>0$, each function $f$ of this class satisfies an estimate of the type $|f(x)| \leq M(1+c x)^{q}$ for $x \geq 0$ with certain constants $M, q>0$. We start with the following observation. 
Lemma 2.1 Let $I \subset[0, \infty)$ be a compact interval. For $f \in W[0, \infty)$, there are constants $M, q>0$, such that

$$
|f(x)-f(y)| \leq M \cdot(1+c x)^{q}
$$

for all $x \geq 0$ and $y \in I$.

Proof For $f \in W[0, \infty)$, there are constants $M_{1}, q>0$ such that $|f(x)| \leq M_{1}(1+c x)^{q}$ for $x \geq 0$. In particular, with $M_{2}:=\max _{t \in I}|f(t)|$, we have $|f(x)-f(y)| \leq M_{1}(1+c x)^{q}$ $+M_{2} \leq M(1+c x)^{q}$ for $x \geq 0$ and $y \in I$ with a certain positive constant $M$.

The following lemma guarantees the convergence of

$$
\sum_{\nu=1}^{\infty} p_{n, \nu}^{[c]}(x) \int_{0}^{\infty} \mu_{n, v, \rho}^{[c]}(t) f(t) d t
$$

in (1.3), for all functions $f \in W[0, \infty)$.

Lemma 2.2 Let $c>0$ and $n, v, q \in \mathbb{N}$. If $f \in W[0, \infty)$ satisfies the estimate $|f(t)|$ $\leq M(1+c t)^{q}$ for $t \in[0, \infty)$, then, for sufficiently large $\rho>0$,

$$
\left|\int_{0}^{\infty} \mu_{n, v, \rho}^{[c]}(t) f(t) d t\right| \leq M \cdot\left(2 \frac{n+c v}{n}\right)^{q} .
$$

Proof As

$\mu_{n, v, \rho}^{[c]}(t)(1+c t)^{q}=\frac{\Gamma\left(\frac{n}{c} \rho+1+v \rho\right) \Gamma\left(\frac{n}{c} \rho+1-q\right)}{\Gamma\left(\frac{n}{c} \rho+1-q+v \rho\right) \Gamma\left(\frac{n}{c} \rho+1\right)}(n \rho+c-q c) p_{n \rho+2 c-q, v \rho-1}^{[c]}(x)$,

we obtain

$$
\left|\int_{0}^{\infty} \mu_{n, v, \rho}^{[c]}(t) f(t) d t\right| \leq M \frac{\Gamma\left(\frac{n}{c} \rho+1+v \rho\right) \Gamma\left(\frac{n}{c} \rho+1-q\right)}{\Gamma\left(\frac{n}{c} \rho+1-q+v \rho\right) \Gamma\left(\frac{n}{c} \rho+1\right)} .
$$

Observing that

$$
\frac{\Gamma\left(\frac{n}{c} \rho+1+v \rho\right) \Gamma\left(\frac{n}{c} \rho+1-q\right)}{\Gamma\left(\frac{n}{c} \rho+1-q+v \rho\right) \Gamma\left(\frac{n}{c} \rho+1\right)} \leq\left(\frac{\frac{n}{c} \rho+v \rho}{\frac{n}{c} \rho-q}\right)^{q}=\left(\frac{\frac{n}{c}+v}{\frac{n}{c}-q / \rho}\right)^{q} \leq\left(2 \frac{n+c v}{n}\right)^{q},
$$

for sufficiently large $\rho>0$, leads to the desired estimate.

Lemma 2.3 Let $c>0, \rho>0$ and $n \in \mathbb{N}$. Then

$$
\frac{\sqrt{2 \pi}}{B\left(\frac{n}{c} \rho+1, v \rho\right)} \leq\left(1+\frac{n}{c v}\right)^{v \rho}\left(1+\frac{c \nu}{n}\right)^{\frac{n}{c} \rho+1 / 2}(v \rho)^{1 / 2} \exp \left(\frac{1}{12 \rho}\right) .
$$

Proof From the definition of the beta function it follows that

$$
\frac{1}{B\left(\frac{n}{c} \rho+1, v \rho\right)}=\frac{v \rho \cdot \Gamma\left(v \rho+\frac{n}{c} \rho+1\right)}{\Gamma(v \rho+1) \Gamma\left(\frac{n}{c} \rho+1\right)} .
$$

By Stirling's formula the gamma function satisfies $\Gamma(z+1)=\sqrt{2 \pi} z^{z+1 / 2} \exp (-z+\vartheta(z))$ with $0<\vartheta(z)<1 /(12 z)$, for $z>0$. Hence,

$$
\frac{\sqrt{2 \pi}}{B\left(\frac{n}{c} \rho+1, v \rho\right)}=\frac{\left(v \rho+\frac{n}{c} \rho\right)^{v \rho+\frac{n}{c} \rho+1 / 2}}{(v \rho)^{v \rho-1 / 2}\left(\frac{n}{c} \rho\right)^{\frac{n}{c} \rho+1 / 2}} \exp \left(\vartheta\left(v \rho+\frac{n}{c} \rho\right)-\vartheta(v \rho)-\vartheta\left(\frac{n}{c} \rho\right)\right)
$$

which implies the desired estimate. 
Lemma 2.4 Let $f \in W[0, \infty), n, N \in \mathbb{N}$ and $1 \leq v \leq N$. For each $\varepsilon>0$ there exist positive constants $T, \rho_{0}$ such that for all $v \in\{1, \ldots, N\}$,

$$
\int_{T}^{\infty} \mu_{n, \nu, \rho}^{[c]}(t)\left|f(t)-f\left(\frac{v}{n}\right)\right| d t \leq \varepsilon \quad\left(\rho \geq \rho_{0}\right) .
$$

Proof Let $1 \leq v \leq N$. By Lemma 2.1 there are positive constants $M$ and $q$ such that $B\left(\frac{n}{c} \rho+1, v \rho\right) \cdot \mu_{n, v, \rho}^{[c]}(t)\left|f(t)-f\left(\frac{v}{n}\right)\right| \leq M(1+c t)^{q} c^{\nu \rho} t^{\nu \rho-1}(1+c t)^{-(n / c+v) \rho-1}$.

Choose $T$ larger than the maximum point $t=\frac{v+q / \rho}{n-c q / \rho}$ (we can assume that $\rho$ is sufficiently large such that $c q / \rho<1$ ) of the unimodal function

$$
(c t)^{v \rho} t^{q}(1+c t)^{-(n / c+v) \rho} .
$$

Then

$$
\begin{aligned}
& B\left(\frac{n}{c} \rho+1, v \rho\right) \int_{T}^{\infty} \mu_{n, \nu, \rho}^{[c]}(t)\left|f(t)-f\left(\frac{v}{n}\right)\right| d t \\
& \leq M \cdot T^{q}(c T)^{v \rho}(1+c T)^{-(n / c+v) \rho} \int_{T}^{\infty} \frac{(1+c t)^{q-1}}{t^{q+1}} d t .
\end{aligned}
$$

Since the integral has a finite value, we derive by applying the estimate in Lemma 2.3 for $1 \leq v \leq N$

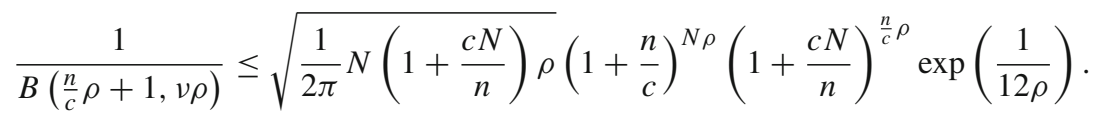

This entails

$$
\begin{aligned}
& \int_{T}^{\infty} \mu_{n, v, \rho}^{[c]}(t)\left|f(t)-f\left(\frac{v}{n}\right)\right| d t \\
& =O(\sqrt{\rho})\left(\left(1+\frac{n}{c}\right)^{N}\left(1+\frac{c N}{n}\right)^{\frac{n}{c}}\left(\frac{c T}{1+c T}\right)^{v}(1+c T)^{-n / c}\right)^{\rho} \\
& =O\left(e^{-\alpha \rho}\right) \quad(\rho \rightarrow \infty)
\end{aligned}
$$

with a positive constant $\alpha$ if $T$ is sufficiently large.

Lemma 2.5 Let $f \in W[0, \infty), n, N \in \mathbb{N}, \delta>0, T>N / n$ and $1 \leq v \leq N$. For each $\varepsilon>0$ there exists a positive constant $\rho_{0}$ such that for all $v \in\{1, \ldots, N\}$,

$$
\int_{\frac{v}{n}+\delta}^{T} \mu_{n, \nu, \rho}^{[c]}(t)\left|f(t)-f\left(\frac{v}{n}\right)\right| d t \leq \varepsilon \quad\left(\rho \geq \rho_{0}\right) .
$$

Proof Let $1 \leq v \leq N$. As in the above proof we have

$$
\begin{aligned}
B & \left(\frac{n}{c} \rho+1, v \rho\right) \int_{\frac{v}{n}+\delta}^{T} \mu_{n, v, \rho}^{[c]}(t)\left|f(t)-f\left(\frac{v}{n}\right)\right| d t \\
& \leq M_{2}\left(c\left(\frac{v}{n}+\delta\right)\right)^{v \rho}\left(1+c\left(\frac{v}{n}+\delta\right)\right)^{-(n / c+v) \rho} \cdot T \max _{\frac{1}{n}+\delta \leq t \leq T} \frac{(1+c t)^{q-1}}{t} .
\end{aligned}
$$

Put

$$
\sigma_{0}=\sigma_{0}(v):=\frac{v}{n} \leq \frac{v}{n}+\delta=: \sigma(v)=\sigma
$$


For a better readability we don't indicate that $\sigma_{0}$ and $\sigma$ depend on $v$. Then

$$
\begin{gathered}
B\left(\frac{n}{c} \rho+1, v \rho\right) \int_{\frac{v}{n}+\delta}^{T} \mu_{n, v, \rho}^{[c]}(t)\left|f(t)-f\left(\frac{v}{n}\right)\right| d t \\
\leq M_{2} n T(1+c T)^{q-1}(c \sigma)^{n \sigma_{0} \rho}(1+c \sigma)^{-\left(n / c+n \sigma_{0}\right) \rho} .
\end{gathered}
$$

From Lemma 2.3 we infer that for $1 \leq v \leq N$

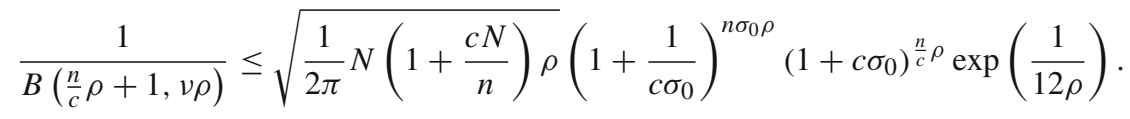

Hence,

$$
\begin{aligned}
& \int_{\frac{v}{n}+\delta}^{T} \mu_{n, v, \rho}^{[c]}(t)\left|f(t)-f\left(\frac{v}{n}\right)\right| d t \\
& \quad \leq O(\sqrt{\rho})\left(1+\frac{1}{c \sigma_{0}}\right)^{n \sigma_{0} \rho}\left(1+c \sigma_{0}\right)^{\frac{n}{c} \rho}(c \sigma)^{n \sigma_{0} \rho}(1+c \sigma)^{-\left(n / c+n \sigma_{0}\right) \rho} \\
& \quad=O(\sqrt{\rho})\left(\frac{1+\frac{1}{c \sigma_{0}}}{1+c \sigma} c \sigma\right)^{n \sigma_{0} \rho}\left(\frac{1+c \sigma_{0}}{1+c \sigma}\right)^{\frac{n}{c} \rho} \quad(\rho \rightarrow \infty) .
\end{aligned}
$$

Now, $\frac{1+\frac{1}{c \sigma_{0}}}{1+c \sigma} c \sigma=1+\frac{1}{\sigma_{0}} \frac{\delta}{1+c \sigma}$ and $\frac{1+c \sigma_{0}}{1+c \sigma}=1-c \frac{\delta}{1+c \sigma}$. This entails,

$$
\int_{\frac{v}{n}+\delta}^{T} \mu_{n, v, \rho}^{[c]}(t)\left|f(t)-f\left(\frac{v}{n}\right)\right| d t=O(\sqrt{\rho})\left[\left(1+\frac{z}{\sigma_{0}}\right)^{\sigma_{0}}(1-c z)^{\frac{1}{c}}\right]^{n \rho}
$$

as $\rho \rightarrow \infty$, where we put $z:=\frac{\delta}{1+c \sigma}$. Furthermore, put $G(z)=\log \left[\left(1+\frac{z}{\sigma_{0}}\right)^{\sigma_{0}}(1-c z)^{\frac{1}{c}}\right]$. Note that $c z=\frac{c \delta}{1+c \sigma}<c \delta<1$. The derivative $G^{\prime}$ satisfies

$$
\begin{aligned}
G^{\prime}(z) & =\frac{d}{d z}\left(\sigma_{0} \log \left(1+\frac{z}{\sigma_{0}}\right)+\frac{1}{c} \log (1-c z)\right) \\
& =\frac{-\left(1+\sigma_{0} c\right) z}{\left(z+\sigma_{0}\right)(1-c z)}<0 \quad \text { for } z \in\left(0, \frac{1}{c}\right) .
\end{aligned}
$$

Therefore, $G$ is monotonically decreasing on $[0,1 / c)$. Because $G(0)=0$, we have $G(z)<0$ for $z \in(0,1 / c)$. Hence,

$$
\max _{1 \leq \nu \leq N}\left(1+\frac{z}{\sigma_{0}}\right)^{\sigma_{0}}(1-c z)^{1 / c}<1 .
$$

Now the lemma follows by (2.1).

Lemma 2.6 Let $f \in W[0, \infty), n, N \in \mathbb{N}, \delta>0$ and $1 \leq v \leq N$. For each $\varepsilon>0$ there exists a positive constant $\rho_{0}$ such that for all $v \in\{1, \ldots, N\}$,

$$
\int_{0}^{\frac{\nu}{n}-\delta} \mu_{n, \nu, \rho}^{[c]}(t)\left|f(t)-f\left(\frac{v}{n}\right)\right| d t \leq \varepsilon \quad\left(\rho \geq \rho_{0}\right) .
$$


Proof Let $1 \leq v \leq N$. As in the proof of Lemma 2.4, we have

$$
\begin{aligned}
& B\left(\frac{n}{c} \rho+1, v \rho\right) \int_{0}^{\frac{v}{n}-\delta} \mu_{n, v, \rho}^{[c]}(t)\left|f(t)-f\left(\frac{v}{n}\right)\right| d t \\
& \leq M_{2} c^{\nu \rho} \int_{0}^{\frac{v}{n}-\delta} t^{\nu \rho-1}(1+c t)^{-(n / c+v) \rho-1+q} d t \\
& \leq M_{2} c^{\nu \rho} \int_{0}^{\frac{v}{n}-\delta} t^{\nu \rho-1}(1+c t)^{-(n / c+v) \rho} d t \cdot \max _{0 \leq t \leq \frac{\nu}{n}-\delta}(1+c t)^{q-1} \\
& \leq M_{2}\left(1+c \frac{N}{n}\right)^{q-1} c^{\nu \rho} \int_{0}^{\frac{v}{n}-\delta} t^{\nu \rho-1}(1+c t)^{-(n / c+v) \rho} d t .
\end{aligned}
$$

Choose $\rho$ so large that the maximum point $\frac{\nu-1 / \rho}{n+c / \rho}$ of $t^{\nu \rho-1}(1+c t)^{-(n / c+v) \rho}$ is larger than $\frac{v}{n}-\delta$. Put

$$
\tau=\tau(\nu):=\frac{v}{n}-\delta \leq \frac{v}{n}=: \tau_{0}(v)=\tau_{0} .
$$

For a better readability we don't indicate that $\tau$ and $\tau_{0}$ depend on $v$. Then

$\int_{0}^{\frac{v}{n}-\delta} \mu_{n, v, \rho}^{[c]}(t)\left|f(t)-f\left(\frac{v}{n}\right)\right| d t \leq O(1) \frac{c^{v \rho}}{B\left(\frac{n}{c} \rho+1, v \rho\right)} \tau \cdot \tau^{n \tau_{0} \rho-1}(1+c \tau)^{-\left(1 / c+\tau_{0}\right) n \rho}$ as $\rho \rightarrow \infty$. By Lemma 2.3 we have

$$
\frac{1}{B\left(\frac{n}{c} \rho+1, v \rho\right)} \leq O(\sqrt{\rho})\left(1+\frac{1}{c \tau_{0}}\right)^{n \tau_{0} \rho}\left(1+c \tau_{0}\right)^{\frac{n}{c} \rho} \exp \left(\frac{1}{12 \rho}\right),
$$

as $\rho \rightarrow \infty$, since $1 \leq v \leq N$. Therefore,

$$
\begin{aligned}
& \int_{0}^{\frac{v}{n}-\delta} \mu_{n, v, \rho}^{[c]}(t)\left|f(t)-f\left(\frac{v}{n}\right)\right| d t \\
& \quad \leq O(\sqrt{\rho})\left[\left(1+\frac{1}{c \tau_{0}}\right)^{\tau_{0}}\left(1+c \tau_{0}\right)^{\frac{1}{c}}(c \tau)^{\tau_{0}}(1+c \tau)^{-\left(1 / c+\tau_{0}\right)}\right]^{n \rho} \\
& \quad=O(\sqrt{\rho})\left[\left(\frac{1+\frac{1}{c \tau_{0}}}{1+c \tau} c\right)^{\tau_{0}}\left(\frac{1+c \tau_{0}}{1+c \tau}\right)^{\frac{1}{c}}\right]^{n \rho}
\end{aligned}
$$

as $\rho \rightarrow \infty$. Now, $\frac{1+\frac{1}{c \tau_{0}}}{1+c \tau} c \tau=1-\frac{1}{\tau_{0}} \frac{\delta}{1+c \tau}$ and $\frac{1+c \tau_{0}}{1+c \tau}=1+c \frac{\delta}{1+c \tau}$. Hence,

$$
\int_{0}^{\frac{v}{n}-\delta} \mu_{n, v, \rho}^{[c]}(t)\left|f(t)-f\left(\frac{v}{n}\right)\right| d t=O(\sqrt{\rho})\left[\left(1-\frac{z}{\tau_{0}}\right)^{\tau_{0}}(1+c z)^{\frac{1}{c}}\right]^{n \rho}
$$

as $\rho \rightarrow \infty$, where we put $z:=\frac{\delta}{1+c \tau}$. Furthermore, put $H(z)=\log \left[\left(1-\frac{z}{\tau_{0}}\right)^{\tau_{0}}(1+c z)^{\frac{1}{c}}\right]$. Note that $z=\frac{\delta}{1+c \tau}<\delta<\frac{1}{n} \leq \frac{v}{n}=\tau_{0}$. The derivative $H^{\prime}$ satisfies

$$
\begin{aligned}
H^{\prime}(z) & =\frac{d}{d z}\left(\tau_{0} \log \left(1-\frac{z}{\tau_{0}}\right)+\frac{1}{c} \log (1+c z)\right) \\
& =\frac{-\left(1+\tau_{0} c\right) z}{\left(\tau_{0}-z\right)(1+c z)}<0, \quad \text { for } z \in\left(0, \tau_{0}\right) .
\end{aligned}
$$


Therefore, $H$ is monotonically decreasing on $\left[0, \tau_{0}\right)$. Because $H(0)=0$, we have $H(z)<0$ for $z \in\left(0, \tau_{0}\right)$. Thus,

$$
\max _{1 \leq \nu \leq N}\left(1-\frac{z}{\tau_{0}}\right)^{\tau_{0}}(1+c z)^{1 / c}<1 .
$$

Now the lemma follows by (2.2).

\section{Proof of the main result}

Proof of Theorem 1.1 Since $\gamma$ is arbitrary, we can assume that there exist a real constant $M>0$ and an integer $q \geq 0$ such that $|f(t)| \leq M(1+c t)^{q}$ for $t \in[0, \infty)$. For $v>b n$ the function $p_{n, v}^{[c]}$ is monotonically increasing on $[0, b]$. In particular, $0 \leq p_{n, v}^{[c]}(x) \leq p_{n, v}^{[c]}(b)$, and Lemma 2.2 implies that

$$
\left|p_{n, \nu}^{[c]}(x) \int_{0}^{\infty} \mu_{n, v, \rho}^{[c]}(t) f(t) d t\right| \leq p_{n, v}^{[c]}(b) \cdot M\left(2 \frac{c v+n}{n}\right)^{q}
$$

for $x \in[0, b]$. Since $p_{n, 0}^{[c]}(x) \leq 1$, we have

$$
\left|\left(B_{n, \rho}^{[c]} f\right)(x)\right| \leq|f(0)|+2^{q} M \sum_{\nu=1}^{\infty} p_{n, v}^{[c]}(b)\left(1+c \frac{v}{n}\right)^{q}<\infty .
$$

The uniform and absolute convergence of the series $B_{n, \rho}^{[c]} f$ allows us to choose an integer $N>b n$ such that

$$
\left|\sum_{\nu=N+1}^{\infty} p_{n, \nu}^{[c]}(x) \int_{0}^{\infty} \mu_{n, v, \rho}^{[c]}(t) f(t) d t\right|<\varepsilon
$$

for sufficiently large $\rho>0$. For the same reason, we can achieve the inequality

$$
\left|\sum_{\nu=N+1}^{\infty} p_{n, v}^{[c]}(x) f\left(\frac{v}{n}\right)\right|<\varepsilon
$$

for all $x \in[0, b]$ and $\rho \geq \rho_{0}$. Therefore,

$$
\left|\left(B_{n, \rho}^{[c]} f\right)(x)-\left(B_{n, \infty}^{[c]} f\right)(x)\right|<\sum_{\nu=1}^{N} p_{n, \nu}^{[c]}(x) \int_{0}^{\infty} \mu_{n, \nu, \rho}^{[c]}(t)\left|f(t)-f\left(\frac{v}{n}\right)\right| d t+2 \varepsilon
$$

for all $x \in[0, b]$ and $\rho \geq \rho_{0}$. From the continuity of the function $f$ there is $\delta>0$ such that $\delta<1 / n$ and $\left|f(t)-f\left(\frac{v}{n}\right)\right|<\varepsilon$ if $\left|t-\frac{v}{n}\right|<\delta$. It follows

$$
\begin{aligned}
& \sum_{\nu=1}^{N} p_{n, \nu}^{[c]}(x) \int_{\frac{v}{n}-\delta}^{\frac{v}{n}+\delta} \mu_{n, \nu, \rho}^{[c]}(t)\left|f(t)-f\left(\frac{v}{n}\right)\right| d t \\
& \quad \leq \varepsilon \sum_{\nu=1}^{N} p_{n, \nu}^{[c]}(x) \int_{0}^{\infty} \mu_{n, \nu, \rho}^{[c]}(t) d t \leq \varepsilon .
\end{aligned}
$$

This inequality and application of the Lemmata 2.4-2.6 leads to the final estimate

$$
\left|\left(B_{n, \rho}^{[c]} f\right)(x)-\left(B_{n, \infty}^{[c]} f\right)(x)\right|<6 \varepsilon
$$

for all $x \in[0, b]$ and $\rho \geq \rho_{0}$. This completes the proof of the theorem. 
Acknowledgements Open Access funding provided by Projekt DEAL.

Open Access This article is licensed under a Creative Commons Attribution 4.0 International License, which permits use, sharing, adaptation, distribution and reproduction in any medium or format, as long as you give appropriate credit to the original author(s) and the source, provide a link to the Creative Commons licence, and indicate if changes were made. The images or other third party material in this article are included in the article's Creative Commons licence, unless indicated otherwise in a credit line to the material. If material is not included in the article's Creative Commons licence and your intended use is not permitted by statutory regulation or exceeds the permitted use, you will need to obtain permission directly from the copyright holder. To view a copy of this licence, visit http://creativecommons.org/licenses/by/4.0/.

\section{References}

1. V.A. Baskakov, An instance of a sequence of positive linear operators in the space of continuous functions. Dokl. Akad. Nauk SSSR 113(2), 249-251 (1957)

2. K. Baumann, M. Heilmann, I. Raşa, Further results for $k$ th order Kantorovich modification of linking Baskakov type operators. Results Math. 69(3), 297-315 (2016). https://doi.org/10.1007/s00025-0150511-X

3. J.L.Durrmeyer, Une formule d'inversion de la transform ée de Laplace: applications à la théorie des moments, Thèse de 3e cycle, Faculté des Sciences de l'Université de Paris (1967)

4. H. Gonska, R. Păltănea, Simultaneous approximation by a class of Bernstein-Durrmeyer operators preserving linear functions. Czechoslovak Math. J. 60(135), 783-799 (2010). https://doi.org/10.1007/ s10587-010-0049-8

5. H. Gonska, R. Păltănea, Quantitative convergence theorems for a class of Bernstein-Durrmeyer operators preserving linear functions. Ukrainian Math. J. 62(7), 1061-1072 (2010). https://doi.org/10.1007/s11253010-0413-8

6. M. Heilmann, Direct and converse results for operators of Baskakov-Durrmeyer operators. Approx. Theory Appl. 5(1), 105-127 (1989). https://doi.org/10.1007/BF02836120

7. M. Heilmann, I. Raşa, $k$-th order Kantorovich modification of linking Baskakov type operators, in P.N. Agrawal et al. (ed.) Recent Trends in Mathematical Analysis and its Applications, Proceedings of the Conference ICRTMAA 2014, Roorkee, India, December 2014, Proceedings in Mathematics \& Statistics, 143, 229-242 (2015)

8. A. Lupaş, Die Folge der Betaoperatoren, Dissertation (Universität Stuttgart, Stuttgart, 1972)

9. S.M. Mazhar, V. Totik, Approximation by modified Szász operators. Acta Sci. Math. 49, 257-269 (1985)

10. R. Păltănea, A class of Durrmeyer type operators preserving linear functions. Ann. Tiberiu Popoviciu Semin. Funct. Equ. Approx. Convexity (Cluj-Napoca) 5, 109-117 (2007)

11. R. Păltănea, Modified Szász-Mirakjan operators of integral form. Carpathian J. Math. 24(3), 378-385 (2008)

12. R. Păltănea, Simultaneous approximation by a class of Szász-Mirakjan operators. J. Appl. Funct. Anal. 9(3-4), 356-368 (2014)

13. R.S. Phillips, An inversion formula for Laplace transforms and semi-groups of linear operators. Ann. Math. (Second Ser.) 59, 325-356 (1954). https://doi.org/10.2307/1969697

14. A. Sahai, G. Prasad, On simultaneous approximation by modified Lupas operators. J. Approx. Theory 45, 122-128 (1985). https://doi.org/10.1016/0021-9045(85)90039-5

15. M. Wagner, Quasi-Interpolanten zu genuinen Baskakov-Durrmeyer-Typ Operatoren, Dissertation Universität Wuppertal (2013)

Publisher's Note Springer Nature remains neutral with regard to jurisdictional claims in published maps and institutional affiliations. 